\title{
Expression changes of cell-cell adhesion-related genes in colorectal tumors
}

\author{
MATEUSZ BUJKO $^{1}$, PAULINA KOBER ${ }^{1}$, MICHAL MIKULA ${ }^{2}$, MARCIN LIGAJ $^{3}$, \\ JERZY OSTROWSKI ${ }^{2}$ and JANUSZ ALEKSANDER SIEDLECKI ${ }^{1}$
}

\author{
Departments of ${ }^{1}$ Molecular and Translational Oncology, ${ }^{2}$ Genetics and ${ }^{3}$ Pathology, \\ Maria Sklodowska-Curie Memorial Cancer Center and Institute of Oncology, Warsaw 02-781, Poland
}

Received September 10, 2014; Accepted February 10, 2015

DOI: $10.3892 / 01.2015 .3107$

\begin{abstract}
Epithelial tissues achieve a highly organized structure due to cell-cell junction complexes. Carcinogenesis is accompanied by changes in cell interactions and tissue morphology, which appear in the early stages of benign tumors and progress along with invasive potential. The aim of the present study was to analyze the changes in expression levels of genes encoding intercellular junction proteins that have been previously identified to be differentially expressed in colorectal tumors compared with normal mucosa samples (fold change, $>2.5$ ) in genome-wide expression profiling. The expression of 20 selected genes was assessed using quantitative reverse transcription polymerase chain reaction in 26 colorectal cancer, 42 adenoma and 24 normal mucosa samples. Between these tissue types, differences were observed in the mRNA levels of genes encoding adherens junction proteins (upregulation of $\mathrm{CDH} 3$ and $\mathrm{CDH11}$, and downregulation of $\mathrm{CDH} 19$ and $P T P R F$ ), tight junction proteins (upregulation of $C L D N 1$ and $C L D N 2$, and downregulation of $C L D N 5, C L D N 8, C L D N 23$, $C L D N 15, J A M 2$ and $C G N$ ) and desmosomes (upregulation of $D S C 3$ and $D S G 3$, and downregulation of $D S C 2$ ), in addition to a decrease in the expression of certain other genes involved in intercellular connections: $P C D H B 14, P C D H 7, M U P C D H$ and NEOI. The differences between tissue types were statistically significant, and separate clustering of normal adenoma and carcinoma samples was observed in a hierarchical clustering analysis. These results indicate that the morphological changes in neoplastic colon tissue that occur during the 'adenoma-carcinoma sequence' are accompanied by specific changes in the expression of multiple genes encoding the majority of cell-cell junction complexes. The particular differential expression
\end{abstract}

Correspondence to: Mr. Mateusz Bujko, Department of Molecular and Translational Oncology, Maria Sklodowska-Curie Memorial Cancer Center and Institute of Oncology, 5 W.K. Roentgena, Warsaw 02-781, Poland

E-mail: mbujko@coi.waw.pl

Key words: colorectal cancer, colorectal adenoma, gene expression, cell-cell adhesion patterns appear to be consistent among patients with cancer and adenoma, in addition to normal mucosa samples.

\section{Introduction}

Epithelial tissue is fundamental to the physiology of organisms. Maintenance of its complex architecture and function requires strict spatial and temporal coordination of various processes (1). Loss of control over the cell cycle, migration and adhesion results in the deregulation of normal tissue function, and may lead to the development of epithelium-related diseases, including cancer (2). Adhesion molecules are therefore of significance in the study of tumor progression, particularly due to their contribution to signal transduction and cell communication, in addition to adhesion (2-6).

Epithelial tissues achieve their highly organized structure through cell-cell junction complexes. Four main types of cell-cell junctions have been identified, which form areas of contact between cells: Adherens junctions, desmosomes, tight junctions and gap junctions $(2,5)$.

Adherens junctions and desmosomes are anchoring-type junctions, which hold cells together allowing them to form certain complex structures. The junctions are formed predominantly by interactions between the extracellular domains of transmembrane cadherin proteins, which are also anchored to the cytoskeleton (3). Tight junctions (or occluding junctions) function as selective barriers for ions and molecules, controlling their passage through adjacent cells. Tight junctions are critical for maintaining cell polarity, and are localized near the apical membrane of the cell $(4,6)$. These junctions are maintained primarily by transmembrane proteins including occludin and a variety of claudins $(4,6)$. Gap junctions are channels made of connexins, which allow cells to communicate via the direct flow of inorganic ions and small water-soluble molecules between the cytoplasm of neighboring cells (5).

Colorectal adenocarcinoma (AC) is among the most common malignancies worldwide, accounting for the diagnosis of $>1.2$ million cases and $>600,000$ mortalities, annually (7). According to the generally accepted model, it develops from the mucosa of colonic crypts through the stage of benign adenoma (AD). The neoplastic progression from colonic mucosa to invasive tumor is accompanied by distinct molecular alterations and changes in morphological 
tissue characteristics, known as the 'adenoma-carcinoma sequence' (1). This involves conformational changes, loss or disruption of cell adhesion and increased cell mobility. These changes appear in the early stages of benign adenomas, and progress concurrently with the invasive potential of tumors. They are also features of the epithelial-mesenchymal transition phenomenon, which is crucial for the development of invasive phenotypes. Dissociation of cell-cell junctions is an important element of these processes $(3,8)$.

Genes encoding proteins involved in intercellular adhesion are differentially expressed between normal colonic mucosa and neoplastic epithelia of adenoma and cancer. In the present study, quantitative reverse transcription polymerase chain reaction (qPCR) was used to assess the expression levels of 20 genes encoding cell-cell adhesion-related proteins with the greatest difference in expression levels between normal and tumor tissue. Candidate genes were selected based on the results of previous microarray transcriptome profiling of normal and neoplastic colorectal tissues (9).

\section{Materials and methods}

Patients. Fresh frozen tumor tissue samples were obtained from 26 cancer patients, with 15 samples of corresponding normal colonic (NC) mucosa sections, and 42 colorectal adenoma samples. Clinical tissue sample sections were collected at the Maria Sklodowska-Curie Memorial Cancer Center and Institute of Oncology (Warsaw, Poland) upon the agreement of the ethics committee of the Maria Sklodowska-Curie Memorial Cancer Center and Institute of Oncology and written informed consent was obtained from each patient.

$\mathrm{AC}$ tissue and adjacent normal colon tissue were obtained following surgical resection through laparotomy. NC sections were collected from a distance of $>5 \mathrm{~cm}$ from the tumor tissue. Colonic AD samples were obtained during colonoscopic polypectomy. Samples of normal mucosa from nine healthy individuals were also collected during colonoscopy. Tissue samples were snap frozen in liquid nitrogen and stored at $-80^{\circ} \mathrm{C}$. Cryostat sections were prepared for each specimen using a Microm HM 505E (Zeiss, Oberkochen, Germany) and upper and lower sections from each cryosection collection were evaluated by a pathologist to control the relative cell type content. Patient characteristics are shown in Table I.

Assessment of gene expression levels. A total of 20 genes encoding molecules involved directly in cell-cell adhesion were selected, based on annotation in the Gene Ontology (GO) database and a literature search of the PubMed database. Genes that exhibited a fold change (FC) in expression of $>2.5$ between normal and tumor tissue, and reached a significance threshold (adjusted P-value $<0.05$ ), based on previous microarray analyses, were included (9).

Total RNA from tissue samples was isolated with the use of RNeasy Mini (Qiagen, Hilden, Germany). From each tissue sample, $1 \mu \mathrm{g}$ of RNA was subjected to reverse transcription with SuperScript II Reverse Transcriptase (Invitrogen Life Technologies, Carlsbad, CA, USA). qPCR was conducted in 384 well plates using an Applied Biosystems 7900HT Fast Real-Time PCR System (Applied Biosystems Life Technologies, Foster City, CA, USA) with Sensimix SYBR kit (Bioline
Table I. Patient characteristics and sample description.

\begin{tabular}{lc}
\hline A, Cancer patients & $\mathrm{n}$ \\
\hline Patients, $\mathrm{n}$ & 26 \\
Gender, $\mathrm{n}$ & \\
Male & 11 \\
Female & 15 \\
Age, years & \\
Range & $38-81$ \\
Median & 64 \\
Astler-Coller stage, $\mathrm{n}$ & \\
B1 & 4 \\
B2 & 15 \\
C2 & 7 \\
Tumor location, $\mathrm{n}$ & \\
Caecum & 4 \\
Ascending & 7 \\
Transverse & 1 \\
Descending & 2 \\
Sigmoid & 6 \\
Rectum & 6 \\
Carcinoma content, $\%$ & \\
Range & $51-98$ \\
Median & 75.5 \\
\hline
\end{tabular}

B, Adenoma patients

Patients, $\mathrm{n}$

Gender, $\mathrm{n}$

Male

Female

Age, years

Range

Median

Adenoma size, $\mathrm{mm}$

Range

Median

Histopathology, n

Tubular adenoma

Tubulovillous adenoma

Polyp location, $\mathrm{n}$

Caecum

Ascending

Transverse

Descending

Sigmoid

2

Rectum

25

Adenoma content, $\%$

Range

Median

C, Normal mucosa samples

Samples, $\mathrm{n}$

Total

24

From cancer patients

From healthy individuals

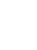


Table II. Primer sequences used in quantitative real-time polymerase chain reaction.

Gene symbol

\section{$\mathrm{CDH} 3$}

CDH11

CDH19

PTPRF

DSC2

DSC 3

DSG3

CLDN1

CLDN2

CLDN5

CLDN8

CLDN15

CLDN23

JAM2

$C G N$

$M U P C D H$

PCDH7

PCDHB14

PCDHB16

NEO1

$A C T B$

Forward primer, 5'-3'

Reverse primer, 5'-3'

CAGTGCTAAACAGAGCTGGC

CAGCAGAAATCCACAATCGG

TGATTCCCTCCAGACCTACG

CTTCTCCACCACCTTCAGC

CTGACCCTCGCGATCTTA

GACCCTCGTGATCTTCAGTC

CCATCTTCGTGGTGGTCATA

CTTCTTGCAGGTCTGGCTAT

GGTTGAATTGCCAAGGATGC

CCTTCCTGGACCACAACATC

CCATGCCTTAGAAATCGCTG

CCATCTTCGAGAACCTCTGG

ACAGGACTCCTTGGACGATT

GTCGCCCTGGGCTATCATAA

CACCATGCTGCAGTTCAAAT

CTTCTACGCAGAGGTTGAGG

GACACGGGGACCATTTACTC

CCAATACGAGGTGTGTCTGA

ATGGATGCACAATCGGAGAC

TACAGAGGGCATGAGTCAGA

AGAGCTACGAGCTGCCTGAC
AGGCTGAAGTGACCTTGGAG

CTTCATAAGGGGCAGCAAAC

AGCGAGGTCCCAACTCATTA

GCCTGGGTGAGATCAACACT

TTGCAGCTGTAAAGCACTCT

ACCTGAAGCACTCTTCCAAA

CCCATTCACGTTTTTGCCTT

AGAGCCTGACCAAATTCGTA

TGAGTCCTGGCTTAAGGGAA

CGAGTCGTACACTTTGCACT

TGTTTTCAATGAAGGCCGAC

CCTGAATATACCCAGAGAGGG

TATGAAAAGCCCGTCACTCC

CTTTGGGGTTTTGCAGGCTA

TCCCTCAGGATGCCATAGAT

GGGCTCCTGTTCGGAAAC

AAAGCGAGACTGTAGCTGTG

TCCCCCATATTCCTACCAGT

CTCCGTTTCTTCCACTACGG

GGGGGTATTTCGAACGGATT

AAGGTAGTTTCGTGGATGCC
Reagents Ltd., London, UK), according to manufacturer's recommendations, in a volume of $5 \mu \mathrm{l}$.

$A C T B$ ( $\beta$-actin) was selected for use as a reference gene following the evaluation of four potential reference genes [ACTB, UBC (Ubiquitin C), RPLPO (60S acidic ribosomal protein P0) and GAPDH (Glyceraldehyde 3-phosphate dehydrogenase)] with GeNorm software (http://medgen.ugent. be/ jvdesomp/genorm/).

All primer sequences are listed in Table II. The standard curves based on the amplification of a known concentration of cDNA template were used for the quantification of PCR products in the samples.

Statistical analysis. The values of expression levels were compared using a two-sided Mann-Whitney U test, and a two-sided Fisher's exact test was used for the analysis of proportion. $\mathrm{P}<0.05$ was considered to indicate a statistically significant difference. Data were analyzed and visualized using GraphPadPrism (La Jolla, CA, USA). A hierarchical clustering analysis was conducted using Cluster 3.0, and the results were visualized using TreeView 1.6 software (Stanford University School of Medicine, Stanford, CA, USA).

\section{Results}

The expression levels of 20 genes encoding proteins involved in cell-cell adhesion were evaluated using qPCR. The genes were selected based on previous microarray transcriptome profiling of colorectal cancer, adenoma and normal tissues, and fulfilled two criteria: They encode molecules directly involved in intercellular junctions, and exhibited a FC of $>2.5$ between cancer and normal, or adenoma and normal tissue. These criteria resulted in the selection of genes encoding components of adherens junctions [CDH3 (cadherin 3), $\mathrm{CDH} 11$ (cadherin 11), CDH19 (cadherin 19) and PTPRF (protein tyrosine phosphatase, receptor type $\mathrm{F}$ )], tight junctions [CLDN1 (claudin 1), CLDN2 (claudin 2), CLDN5 (claudin 5), CLDN8 (claudin 8), CLDN15 (claudin 15), CLDN23 (claudin 23), CGN (cingulin) and JAM2 (junctional adhesion molecule 2)] and desmosomes [DSC2 (desmocollin 2), DSC3 (desmocollin 3) and DSG3 (desmoglein 3)], in addition to certain other surface molecules: $P C D H 7$ (protocadherin 7), $M U P C D H$ (mucin like protocadherin), $P C D H B 14$ (protocadherin beta 14), $P C D H B 16$ (protocadherin beta 16) and NEOI (neogenin). Although other adhesion-related genes, including those encoding for gap junction proteins, have been demonstrated by microarray studies to differ in expression levels between tissue types, they did not meet the FC criterion.

Among the genes encoding adherens junction components, expression of cadherin genes $\mathrm{CDH} 3$ and $\mathrm{CDH} 11$ was markedly increased in tumor tissue compared with normal tissue. This was accompanied by a decrease in the expression of CDH19 and PTPRF, a gene that encodes a tyrosine phosphatase associated with adherens junctions (Fig. 1A).

In tumor samples (AC and $\mathrm{AD}$ tissue), six claudin genes, encoding the crucial extracellular elements of tight junctions, were aberrantly expressed in comparison with normal tissue. The changes included the upregulation of CLDN1 and $C L D N 2$, and the downregulation of CLDN5, CLDN8, $C L D N 15$ and $C L D N 23$. A decrease in the expression of $C G N$ and $J A M 2$, which encode other tight junction proteins, was also observed (Fig. 1B). In addition, tumor tissues were 
A Adherence junctions genes
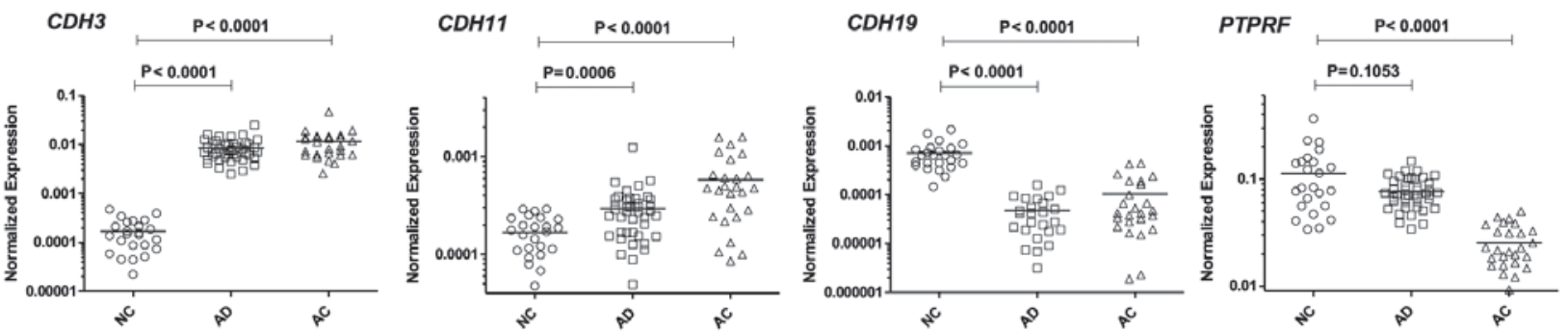

B Desmosome genes
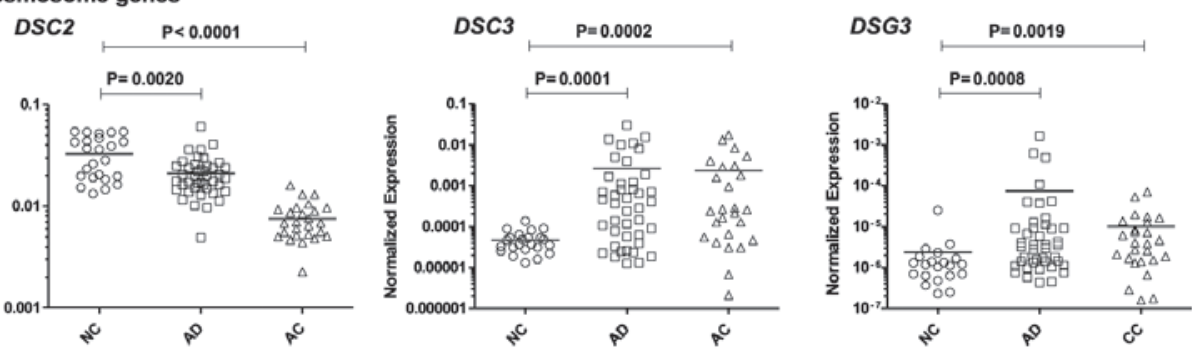

C
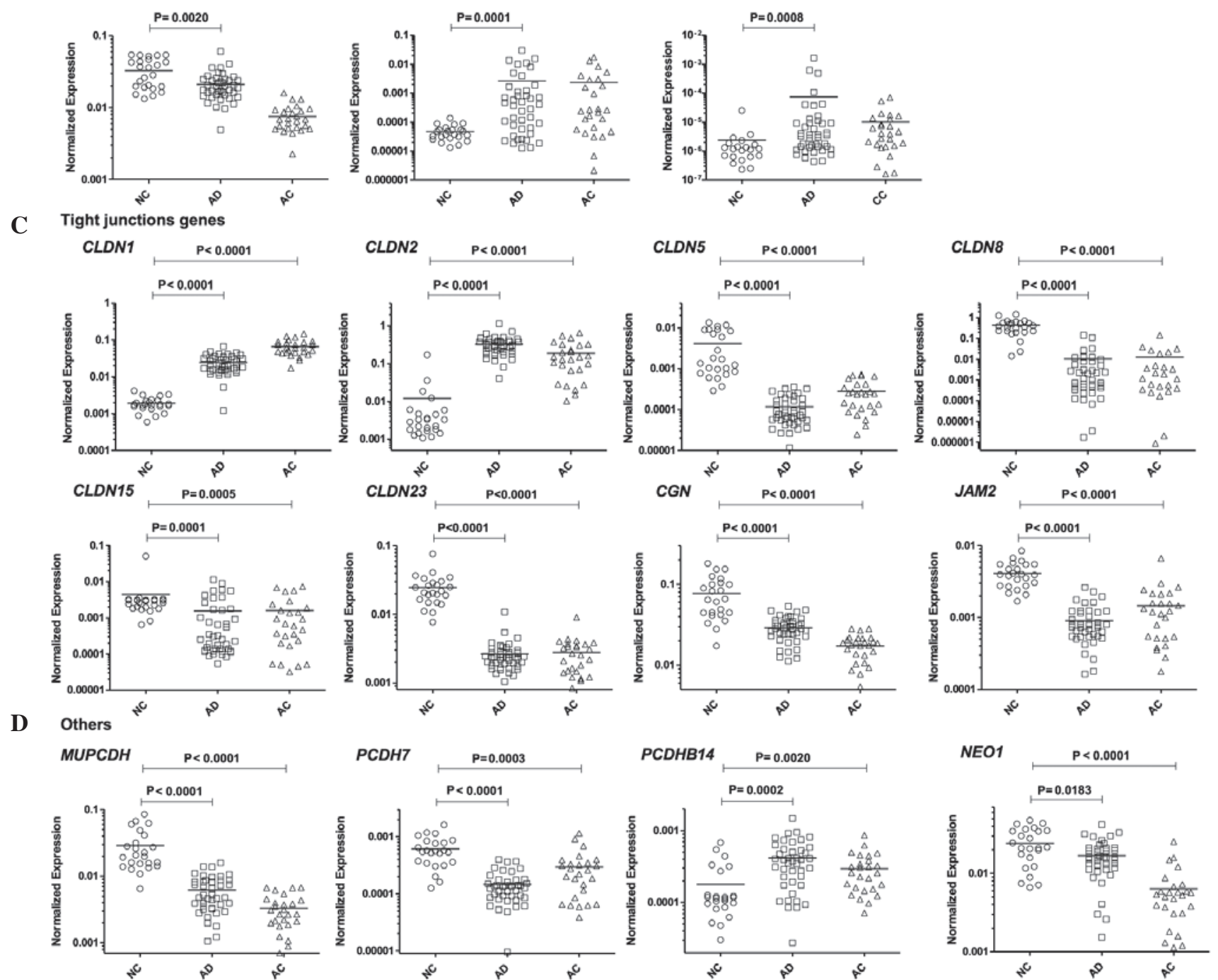

Figure 1. Analysis of mRNA expression levels of selected (A) adherence, (B) desmosome, (C) tight junction and (D) 'other' genes. P-value refers to the Mann-Whitney test. NC, normal colonic mucosa; AD, adenoma; AC, adenocarcinoma. Horizontal bars indicate mean value.

characterized by the altered expression of DSC2 (downregulated), DSC3 (upregulated) and DSG3 (upregulated), encoding desmosome elements (Fig. 1C). Compared with $\mathrm{NC}$, the protocadherin genes $P C D H 7$ and $M U P C D H$ exhibited lower expression in the two tumor tissue types, whilst $P C D H B 14$ exhibited increased mRNA levels in tumors. Decreased expression of the $\mathrm{NEOI}$ gene, encoding an immunoglobulin superfamily member, was also detected (Fig. 1D). The expression level of one gene, $P C D H B 16$ did not exhibit a significant difference between $\mathrm{NC}$ and tumor tissue, in contrast with the differences observed in microarray experiments ( $\mathrm{NC}$ vs. $\mathrm{AC}, \mathrm{P}=0.0623$; $\mathrm{NC}$ vs $\mathrm{AD}, \mathrm{P}=0.3016$ ). Details of the gene expression comparison are shown in Table III and Fig. 1.

The results of the mRNA expression assessment of the 19 genes that significantly differed between the three types of tissue in the 83 tissue samples were subjected to hierarchical clustering analysis; the selected genes may be divided into three categories based on these results (Fig. 2). The first category comprises genes that are overexpressed in the two neoplastic tissues compared with NC. These genes are progressively 
Table III. Description of the studied genes and results of quantitative polymerase chain reaction analysis, presented as the fold change in expression levels in normal mucosa versus adenoma, and normal mucosa versus cancer tissue.

\begin{tabular}{|c|c|c|c|c|}
\hline $\begin{array}{l}\text { Gene } \\
\text { symbol }\end{array}$ & Encoded protein & Protein function & $\begin{array}{l}\mathrm{NC} \text { vs. AD, } \\
\text { fold-change }\end{array}$ & $\begin{array}{l}\mathrm{NC} \text { vs. AC, } \\
\text { fold-change }\end{array}$ \\
\hline $\mathrm{CDH3}$ & P-cadherin & Adherent junction formation & 50.02 & 68.91 \\
\hline $\mathrm{CDH} 11$ & OB-cadherin & Adherent junction formation & 1.74 & 3.43 \\
\hline $\mathrm{CDH} 19$ & Cadherin 19 & Adherent junction formation & -31.41 & -8.13 \\
\hline PTPRF & Protein tyrosine phosphatase, receptor type F & Adherent junction regulation & $-1.48^{\mathrm{a}}$ & -4.42 \\
\hline DSC2 & Desmocollin 2 & Epithelial desmosomal cadherin & $-1.56^{\mathrm{a}}$ & -4.37 \\
\hline DSC3 & Desmocollin 3 & Desmosome forming cadherin & 55.75 & 49.40 \\
\hline DSG3 & Desmoglein 3 & Desmosome forming cadherin & 31.62 & $4.28^{\mathrm{a}}$ \\
\hline$C L D N 1$ & Claudin 1 & Structural tight junction component & 12.96 & 35.17 \\
\hline$C L D N 2$ & Claudin 2 & Structural tight junction component & 26.94 & 15.62 \\
\hline$C L D N 5$ & Claudin 5 & Structural tight junction component & -35.64 & -14.86 \\
\hline CLDN8 & Claudin 8 & Structural tight junction component & -41.16 & -33.81 \\
\hline CLDN15 & Claudin 15 & Structural tight junction component & -2.87 & -2.76 \\
\hline CLDN23 & Claudin 23 & Structural tight junction component & -9.36 & -8.87 \\
\hline$J A M 2$ & Junctional adhesion molecule B & Structural tight junction component & -4.57 & -2.85 \\
\hline$C G N$ & Cingulin & $\begin{array}{l}\text { Intracellular tight junction element, } \\
\text { adhesion related signaling }\end{array}$ & -2.66 & -4.49 \\
\hline$M U P C D H$ & Mu-protocadherin & Cadherin family protein & -4.63 & -8.70 \\
\hline PCDH7 & Protocadherin-7 & Cell-cell adhesion & 4.22 & 2.01 \\
\hline PCDHB14 & Protocadherin beta-14 & Homophillic cell-cell connections & 2.34 & 1.64 \\
\hline PCDHB16 & Protocadherin beta-16 & Homophillic cell-cell connections & $1.91^{\mathrm{a}}$ & $1.87^{\mathrm{a}}$ \\
\hline NEO1 & Neogenin & $\begin{array}{l}\text { Cell-cell adhesion, cell growth } \\
\text { and differentiation }\end{array}$ & $-1.44^{\mathrm{a}}$ & -3.84 \\
\hline
\end{tabular}

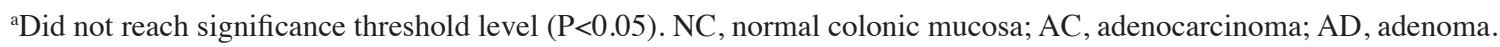

upregulated throughout the stages of cancer progression from NC to $\mathrm{AC}(C D H 3, C D H 11, C L D N 1)$, show comparable expression in $\mathrm{AD}$ and $\mathrm{AC}$, or exhibit the highest mRNA levels in AD (DSC3, DSG3, CLDN2 and PCDHB14). The second category is composed of genes that undergo gradual downregulation during the progression from NC to AC: $C G N, M U P C D H$, DSC2, PTPRF and NEO1. The third category consists of genes that exhibited lower expression in the two neoplastic tissue types, with comparable expression in $\mathrm{AD}$ and $\mathrm{AC}$ (CLDN8, $C L D N 15, C L D N 23)$, as well as those with lowest expression levels in $\mathrm{AD}(P C D H 7, J A M 2$ and CLDN5).

The clustering analysis clearly distinguished tumor samples from normal mucosa. Cancer patients (with exception of two samples) were grouped in one cluster, which also included 12 AD samples. Notably, these patients did not differ from the remaining AD patients (grouped in a second cluster) with regard to clinically relevant features, including dysplasia grade, AD size or histopathological subtype. The only observed difference was in the location of the tumor: Of the 12 adenoma samples that were clustered with the AC samples, 6 samples were located in the transverse and descending colon, while the majority of the remaining ADs, clustered in the second category, were located in the sigmoid colon and rectal regions (25/30). However, the difference in proportions was only marginally below the significance threshold level $(\mathrm{P}=0.0491$, two-sided Fisher's exact test $)$.
Two types of NC samples were used: Sections of normal epithelium adjacent $(>5 \mathrm{~cm})$ to $\mathrm{AC}(\mathrm{NC}-\mathrm{A})$ as well as tissue from healthy individuals obtained during colonoscopy (NC-K). Each of these sample types clustered together, however, both types exhibited similar patterns of expression.

\section{Discussion}

The expression profile of junctional proteins is essential for the maintenance of epithelial tissue architecture, and its disruption in tumors results in the dissociation of adhesion complexes. This is a hallmark of carcinogenesis-related morphological changes, including the epithelial-mesenchymal transition of neoplastic tissue, a crucial step in tumor progression (8).

A number of studies have investigated the role of aberrant expression of a single or small number of a priori selected cell-cell adhesion-related genes in colorectal tumors. Cadherin genes are the most extensively studied, particularly E-cadherin (3), however, genes encoding for tight junction (4) proteins, desmosomes (10) and gap junction (5) proteins have also been investigated. The present study focused on the mRNA expression levels of intercellular adhesion-related genes that had previously been identified to differ to the greatest extent between normal mucosa and colorectal tumor tissues in a microarray transcriptome study (9). In total, 20 genes that fulfilled the inclusion criteria were selected, and 


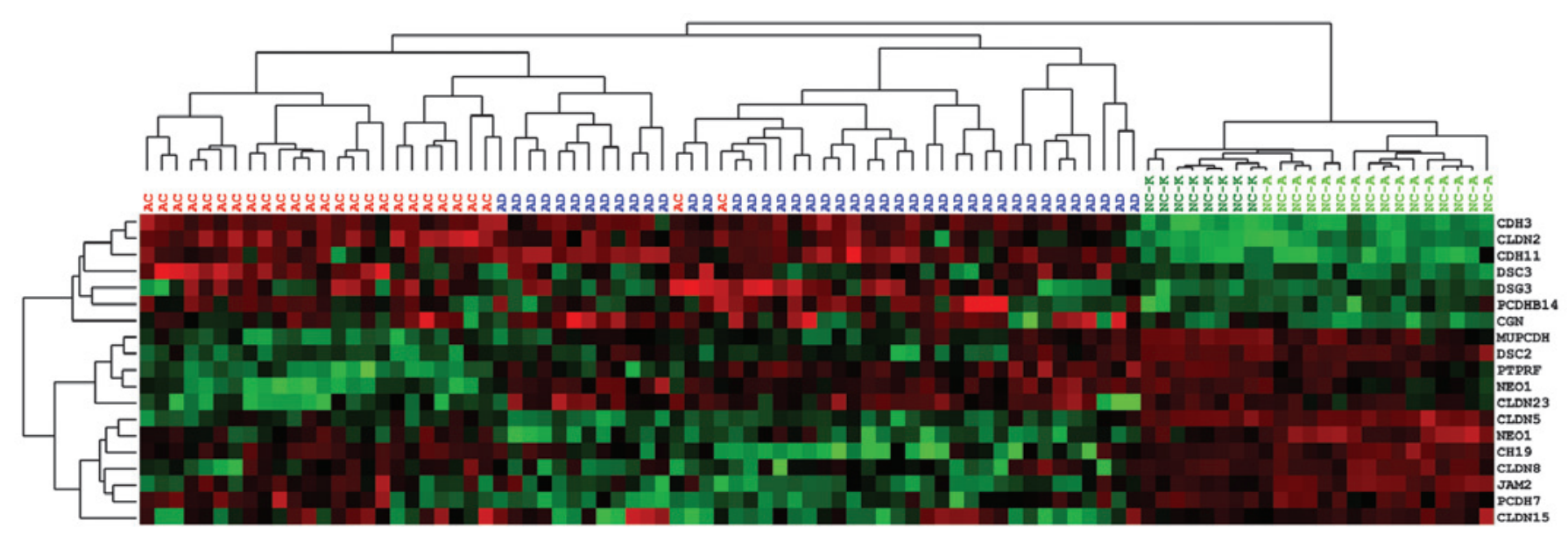

Figure 2. Hierarchical clustering of mRNA expression data from qPCR analysis of the studied intercellular adhesion genes. Red indicates expression levels greater than the mean, and green indicates expression levels lower than the mean. qPCR, quantitative polymerase chain reaction. AC, adenocarcinoma (red); $\mathrm{AD}$, adenoma (dark blue); NC-A normal colon epithelium adjacent $(>5 \mathrm{~cm}$ ) to AC (light green); NC-K normal colon epithelium from healthy individuals (dark green).

their expression levels were evaluated using qPCR, followed by an analysis of the association between gene expression and patient characteristics. The majority of these genes encode components of three basic intercellular complexes: Adherens junctions, desmosomes and tight junctions.

Three genes encoding adherens junction cadherins $(\mathrm{CDH} 3$, $C D H 11$ and $C D H 19$ ) were included in qPCR evaluation. Notably, the E-cadherin gene (the best characterized cadherin in AC) was identified among genes that differ in expression between $\mathrm{NC}$ and $\mathrm{AC}$ tissue, however, it was not among the genes with the highest $\mathrm{FC}$ in expression between these tissue types.

A marked increase in $\mathrm{CDH} 3$ (P-cadherin) expression was detected in tumors, compared with NC. P-cadherin is predominantly expressed in the stratified squamous epithelia, including the esophagus. Its expression is limited to the basal cell layer, and is not observed in the mucosa of the colon or rectum (11). The role of P-cadherin in colorectal carcinogenesis has not been extensively studied, however, its increased expression has previously been reported based on immunohistochemical staining of AD (12) and AC (13). P-cadherin is classified as an oncogene due to its ability to increase crypt cell proliferation in the context of inflammation (14). In addition, two reports have suggested that $\mathrm{CDH} 3$ upregulation in cancer tissue is associated with gene promoter demethylation $(14,15)$. However, using a quantitative approach, increased promoter hypomethylation was identified in only $77 \%$ of carcinomas (15), which does not account for the universal upregulation of the expression of this gene in $\mathrm{AD}$ and cancer. P-cadherin has also been used as a target for immunotherapy in the treatment of AC (16).

CDH11 encodes OB-cadherin, which is expressed predominantly in osteoblastic cells. Its function in tumorigenesis has been investigated in gliomas, where it acts as a marker of the mesenchymal phenotype (17), as well as in prostate (18) and breast cancer (19), where its expression is important in metastasis. Recently, the overexpression of $\mathrm{CDH} 11$ was identified as a marker of cancer-associated stromal cells, and this stromal expression was found to have prognostic value (20). Increased immunohistochemical expression was also observed in $\mathrm{AC}$ epithelial cells (20). It has been hypothesized that, in certain tumors or tumor cell lines, $C D H 11$ acts as a suppressor gene, and its expression is downregulated via a DNA methylation mechanism $(21,22)$.

Little has been established with regard to $\mathrm{CDH} 19$ in tumors. The highest expression of this gene is observed in heart tissue and melanocytes (according to the Genevestigator database) (23), and deletion of the 18q22 region that comprises $C D H 19$, in addition to a decrease in gene expression, have been identified in head and neck tumors (24). The results of the present study suggest that it may be important in colorectal tumor development and progression. However, as the function of the encoded cadherin has not been fully elucidated, this observation requires further investigation.

A marked decrease in PTPRF expression in tumor tissue, compared with $\mathrm{NC}$, was observed in the present study, which has not previously been reported. This gene belongs to the large family of receptor-associated protein phosphatases, which negatively regulate receptor-associated kinases (25). PTPRF is localized to adhesion junctions and is important in E-cadherin and $\beta$-catenin signaling (26-28). The role of PTRPF in E-cadherin signaling, in addition to its high expression in NC tissue (according to the Genevestigator database) (23) and the identification of mutations of this gene in AC (29), suggests that a reduction in tyrosine phosphatase may be significant in adherens junction disintegration.

The comparison of $\mathrm{NC}$ and tumor tissue revealed aberrant expression of desmosome-specific cadherins DSC2 and DSC3. Desmocollin 2 is the major extracellular component of demosomes in normal colorectal mucosa. The downregulation of this gene has been observed in cancer tissue, and is associated with shortened survival of patients $(10,30)$. DSC2 downregulation is accompanied by the de novo expression of DSC3; this transition has been called 'desmosome switching', similarly to the well-described 'cadherin switching' process (10). This phenomenon has not been fully described, however, it is concurrent with the observations of the current study, which identified the equivalent directional changes in DSC2 and DSC3 expression. The increased expression of $D S G 3$, encoding the extracellular desmosome protein desmoglein, also appears to be involved in 
desmosome remodeling, which has not previously been reported. As desmosomes contribute to strong intercellular adhesion, and exhibit differential expression between normal and tumor tissue, the role of desmosomes in AC may be of greater significance than has previously been predicted.

Aberrant expression of genes encoding tight junction proteins, particularly those belonging to claudin family, were observed in the present study. Claudins, along with occludin, are essential structural elements of tight junctions, and constitute a family of 24 proteins that are important in carcinogenesis (6). The results of the current study were consistent with previous reports of upregulation of CLDN1 and CLDN2 (31-34), and downregulation of CLDN8 (34) and CLDN23 (35) in tumor tissue compared with NC. A decrease in CLDN5 and CLDN15 levels was also identified in colorectal $A D$ and $A C$, that had not previously been described. Claudin -5 is primarily detected in the brain and endothelium, and is also present in epithelial cells, including those of intestine (36), and in certain cancers (37). In addition, this protein is involved as a structural component in the junctions between tumor epithelial and vascular endothelial cells, and results from in vitro experiments in HECV endothelial cells indicate that it contributes to cell motility and angiogenic potential, which are reduced when CLDN5 is overexpressed (38). Claudin-5 is also reported to be associated with neoangiogenesis in ovarian cancer (39). The decrease of CLDN5 expression in tumor tissue, as observed in the present study, may reflect changes in tight junctions between cells, and functional changes in tumor vascularization. A decrease in CLDN5 expression has also been observed in Crohn's disease, an inflammatory bowel disease that exhibits a variety of mucosal changes, including preneoplastic lesions, which leads to an increased risk of colorectal cancer (40).

Less is known about the role of CLDN15 in tumors. In mice, claudin-15 was detected among claudins with the highest expression in the colon (41) and it exhibited altered expression in the rat models of colitis and colitis-associated cancer (42). The current study also confirmed the differential expression between AC/AD and NC of two other genes encoding tight junction components: $J A M 2$, a gene that has been found to be hypermethylated in AC (43), and $C G N$. The latter encodes an intracellular tight junction protein, and its overexpression in $\mathrm{AC}$ has been reported in one immunohistochemical study (44). The function of the encoded protein, cingulin remains elusive; however it appears to be important for junction formation and also negatively regulates cell proliferation (45). Additionally, CGN is downregulated during the epithelial-mesenchymal transition in breast cancer model cells (46). These previous findings indicate that cingulin may suppress tumor development, in accordance with the results of the current study.

As well as the genes associated with the large functional complexes described above, four additional cell-cell adhesion-related genes were aberrantly expressed in the two tumor tissue types compared with NC. These included the following protocadherins: $M U P C D H$, for which downregulation has previously been observed in $\mathrm{AC} \mathrm{(47);} \mathrm{PCDH7}$, which has not been investigated in $\mathrm{AC}$ or $\mathrm{AD}$, but has been found to be methylated in bladder cancer (48); $P C D H B 14$, a member of protocadherin- $\beta$ gene cluster for which function is poorly understood; and NEOI, encoding the neogenin protein which is a member of the immunoglobulin superfamily and has been demonstrated to be downregulated in colorectal AD and $\mathrm{AC}$ (49).

Hierarchical clustering of patients showed that clinical samples are grouped according to the expression levels of the studied genes in two main clusters: NC samples and tumors (AD and AC). Normal mucosa samples share a similar expression pattern whether obtained from cancer patients or from healthy patients. Tumors are further divided into two clusters: one primarily comprises AC samples, while the second is predominantly composed of AD samples. Notably, a portion of AD samples was clustered with the AC samples; tumor location appears to be the only feature that distinguishes these samples from the remaining AD samples. This observation is concordant with previously reported localization-related biological profiles of colon and rectal tumors $(50,51)$.

In conclusion, the current study indicates that morphological changes that occur during in neoplastic colon tissue progression through the 'adenoma-carcinoma sequence' are accompanied by aberrant expression of multiple genes encoding cell-cell adhesion proteins, particularly those of junctional complexes. A number of these genes were previously reported to be involved in the pathogenesis of colorectal $\mathrm{AC}$, however, the altered expression of several genes, including $C D H 19, P T P R F$, DSG3, CLDN5, CLDN15, CGN, PCDH7 and PCDHB14 have been demonstrated for the first time in colorectal neoplasms (compared with NC). The specific differential patterns of gene expression appear to be similar among patients with cancer and adenoma, as well as normal mucosa samples. The main limitation of the present study was that the expression of the selected genes were only investigated at the mRNA level. Thus, further studies using immunohistochemistry and western blot analysis are required to validate these results at the protein level, however, this remains a challenge for multiple gene signatures. Protein analysis would be particularly valuable for genes which have not been investigated previously.

We hypothesize that the tumor-specific, aberrant expression of the aforementioned genes may have practical implications for the future and may serve as novel prognostic markers or provide rationale for novel therapeutic strategies.

\section{Acknowledgements}

This work was supported by a research grant from Ministry of Science and Higher Education of Poland (grant no. IP2011 005471).

\section{References}

1. Ponz de Leon M and Di Gregorio C: Pathology of colorectal cancer. Dig Liver Dis 33: 372-388, 2001.

2. Le Bras GF, Taubenslag KJ and Andl CD: The regulation of cell-cell adhesion during epithelial-mesenchymal transition, motility and tumor progression. Cell Adh Migr 6: 365-373, 2012.

3. Tsanou E, Peschos D, Batistatou A, Charalabopoulos A and Charalabopoulos $\mathrm{K}$. The E-cadherin adhesion molecule and colorectal cancer. A global literature approach. Anticancer Res 28: 3815-3826, 2008.

4. Wang X, Tully O, Ngo B, Zitin M and Mullin JM: Epithelial tight junctional changes in colorectal cancer tissues. ScientificWorldJournal 11: 826-841, 2011.

5. Kańczuga-Koda L: Gap junctions and their role in physiology and pathology of the digestive tract. Postepy Hig Med Dosw (Online) 58: 158-165, 2004 (In Polish). 
6. Turksen K and Troy TC: Junctions gone bad: claudins and loss of the barrier in cancer. Biochim Biophys Acta 1816: 73-79, 2011.

7. Brenner H, Kloor M and Pox CP: Colorectal cancer. Lancet 383: $1490-1502,2014$

8. Sipos F and Galamb O: Epithelial-to-mesenchymal and mesenchymal-to-epithelial transitions in the colon. World $\mathbf{J}$ Gastroenterol 18: 601-608, 2012.

9. Skrzypczak M, Goryca K, Rubel T, et al: Modeling oncogenic signaling in colon tumors by multidirectional analyses of microarray data directed for maximization of analytical reliability. PLoS One 5: e13091, 2010.

10. Khan K, Hardy R, Haq A, Ogunbiyi O, Morton D and Chidgey M: Desmocollin switching in colorectal cancer. Br J Cancer 95: 1367-1370, 2006.

11. Shimoyama Y, Yoshida T, Terada M, et al: Molecular cloning of a human $\mathrm{Ca} 2+$-dependent cell-cell adhesion molecule homologous to mouse placental cadherin: its low expression in human placental tissues. J Cell Biol 109: 1787-1794, 1989.

12. Hardy RG, Tselepis C, Hoyland J, et al: Aberrant P-cadherin expression is an early event in hyperplastic and dysplastic transformation in the colon. Gut 50: 513-519, 2002.

13. Kamposioras K, Konstantara A, Kotoula V, et al: The prognostic significance of WNT pathway in surgically-treated colorectal cancer: $\beta$-catenin expression predicts for disease-free survival Anticancer Res 33: 4573-4584, 2013.

14. Milicic A, Harrison LA, Goodlad R, et al: Ectopic expression of P-cadherin correlates with promoter hypomethylation early in colorectal carcinogenesis and enhanced intestinal crypt fission in vivo. Cancer Res 68: 7760-7768, 2008.

15. Hibi K, Goto T, Mizukami H, et al: Demethylation of the CDH3 gene is frequently detected in advanced colorectal cancer. Anticancer Res 29: 2215-2217, 2009.

16. Yoshioka $\mathrm{H}$, Yamamoto $\mathrm{S}$, Hanaoka $\mathrm{H}$, et al: In vivo therapeutic effect of $\mathrm{CDH} 3 / \mathrm{P}$-cadherin-targeting radioimmunotherapy. Cancer Immunol Immunother 61: 1211-1220, 2012

17. Kaur H, Phillips-Mason PJ, Burden-Gulley SM, et al: Cadherin-11, a marker of the mesenchymal phenotype, regulates glioblastoma cell migration and survival in vivo. Mol Cancer Res 10: 293-304, 2012.

18. Chu K, Cheng CJ, Ye X, et al: Cadherin-11 promotes the metastasis of prostate cancer cells to bone. Mol Cancer Res 6 : 1259-1267, 2008.

19. Tamura D, Hiraga T, Myoui A, Yoshikawa H and Yoneda T: Cadherin-11-mediated interactions with bone marrow stromal/osteoblastic cells support selective colonization of breast cancer cells in bone. Int J Oncol 33: 17-24, 2008

20. Torres S, Bartolomé RA, Mendes M, et al: Proteome profiling of cancer-associated fibroblasts identifies novel proinflammatory signatures and prognostic markers for colorectal cancer. Clin Cancer Res 19: 6006-6019, 2013.

21. Li L, Ying J, Li H, et al: The human cadherin 11 is a pro-apoptotic tumor suppressor modulating cell stemness through Wnt/ $\beta$-catenin signaling and silenced in common carcinomas. Oncogene 31: 3901-3912, 2012.

22. Carmona FJ, Villanueva A, Vidal A, et al: Epigenetic disruption of cadherin-11 in human cancer metastasis. J Pathol 228:230-240, 2012.

23. Hruz T, Laule O, Szabo G, et al: Genevestigator v3: a reference expression database for the meta-analysis of transcriptomes. Adv Bioinformatics 2008: 420747, 2008

24. Blons H, Laccourreye O, Houllier AM, et al: Delineation and candidate gene mutation screening of the $18 \mathrm{q} 22$ minimal region of deletion in head and neck squamous cell carcinoma. Oncogene 21: 5016-5023, 2002.

25. Chernoff J: Protein tyrosine phosphatases as negative regulators of mitogenic signaling. J Cell Physiol 180: 173-81, 1999.

26. Serra-Pagès C, Kedersha NL, Fazikas L, et al: The LAR transmembrane protein tyrosine phosphatase and a coiled-coil LAR-interacting protein co-localize at focal adhesions. EMBO J 14: 2827-2838, 1995.

27. Symons JR, LeVea CM and Mooney RA: Expression of the leucocyte common antigen-related (LAR) tyrosine phosphatase is regulated by cell density through functional E-cadherin complexes. Biochem J 365: 513-519, 2002.

28. Bonvini P, An WG, Rosolen A, et al: Geldanamycin abrogates ErbB2 association with proteasome-resistant beta-catenin in melanoma cells, increases beta-catenin-E-cadherin association, and decreases beta-catenin-sensitive transcription. Cancer Res 61: 1671-1677, 2001.
29. Wang Z, Shen D, Parsons DW, et al: Mutational analysis of the tyrosine phosphatome in colorectal cancers. Science 304: 1164-1166, 2004.

30. Knösel T, Chen Y,Hotovy S, Settmacher U, Altendorf-Hofmann A and Petersen I: Loss of desmocollin 1-3 and homeobox genes PITX1 and CDX2 are associated with tumor progression and survival in colorectal carcinoma. Int J Colorectal Dis 27: 1391-1399, 2012.

31. Singh AB, Sharma A, Smith JJ, et al: Claudin-1 up-regulates the repressor ZEB-1 to inhibit E-cadherin expression in colon cancer cells. Gastroenterology 141: 2140-2153, 2011.

32. Huo Q, Kinugasa T, Wang L, et al: Claudin-1 protein is a major factor involved in the tumorigenesis of colorectal cancer. Anticancer Res 29: 851-857, 2009.

33. Kinugasa T, Huo Q, Higashi D, et al: Selective up-regulation of claudin-1 and claudin-2 in colorectal cancer. Anticancer Res 27: 3729-3734, 2007.

34. Gröne J, Weber B, Staub E, et al: Differential expression of genes encoding tight junction proteins in colorectal cancer: frequent dysregulation of claudin-1, -8 and -12 . Int J Colorectal Dis 22: 651-659, 2007.

35. Pitule P, Vycital O, Bruha J, et al: Differential expression and prognostic role of selected genes in colorectal cancer patients. Anticancer Res 33: 4855-4865, 2013.

36. Rahner C, Mitic LL and Anderson JM: Heterogeneity in expression and subcellular localization of claudins $2,3,4$, and 5 in the rat liver, pancreas, and gut. Gastroenterology 120: 411-422, 2001.

37. Soini Y: Expression of claudins 1,2,3,4,5 and 7 in various types of tumours. Histopathology 46: 551-560, 2005.

38. Escudero-Esparza A, Jiang WG and Martin TA: Claudin-5 participates in the regulation of endothelial cell motility. Mol Cell Biochem 362: 71-85, 2012.

39. Herr D, Sallmann A, Bekes I, et al: VEGF induces ascites in ovarian cancer patients via increasing peritoneal permeability by downregulation of Claudin 5. Gynecol Oncol 127: 210-216, 2012

40. Zeissig S, Bürgel N, Günzel D, et al: Changes in expression and distribution of claudin 2,5 and 8 lead to discontinuous tight junctions and barrier dysfunction in active Crohn's disease. Gut 56: 61-72, 2007.

41. Fujita H, Chiba H, Yokozaki H, et al: Differential expression and subcellular localization of claudin-7, $-8,-12,-13$, and -15 along the mouse intestine. J Histochem Cytochem 54: 933-944, 2006.

42. Arimura Y, Nagaishi K and Hosokawa M: Dynamics of claudins expression in colitis and colitis-associated cancer in rat. Methods Mol Biol 762: 409-425, 2011.

43. Oster B, Thorsen K, Lamy $\mathrm{P}$, et al: Identification and validation of highly frequent $\mathrm{CpG}$ island hypermethylation in colorectal adenomas and carcinomas. Int J Cancer 129: 2855-2866, 2011.

44. Citi S, Amorosi A, Franconi F, Giotti A and Zampi G: Cingulin, a specific protein component of tight junctions is expressed in normal and neoplastic human epithelial tissues. Am J Pathol 138: 781-789, 1991.

45. Guillemot L and Citi S: Cingulin regulates claudin-2 expression and cell proliferation through the small GTPase RhoA. Mol Biol Cell 17: 3569-3577, 2006

46. Papageorgis P, Lambert AW, Ozturk S, et al: Smad signaling is required to maintain epigenetic silencing during breast cancer progression. Cancer Res 70: 968-978, 2010.

47. Losi L, Parenti S, Ferrarini F, et al: Down-regulation of $\mu$-protocadherin expression is a common event in colorectal carcinogenesis. Hum Pathol 42: 960-971, 2011.

48. Beukers W, Hercegovac A, Vermeij M, et al: Hypermethylation of the polycomb group target gene PCDH7 in bladder tumors from patients of all ages. J Urol 190: 311-316, 2013.

49. Li VS, Yuen ST, Chan TL, et al: Frequent inactivation of axon guidance molecule RGMA in human colon cancer through genetic and epigenetic mechanisms. Gastroenterology 137: 176-187, 2009.

50. Yamauchi M, Morikawa T, Kuchiba A, et al: Assessment of colorectal cancer molecular features along bowel subsites challenges the conception of distinct dichotomy of proximal versus distal colorectum. Gut 61: 847-854, 2012.

51. Koestler DC, Li J, Baron JA, et al: Distinct patterns of DNA methylation in conventional adenomas involving the right and left colon. Mod Pathol 27: 145-155, 2014. 